\title{
The Security Screening Process Optimization based on Cellular Automatan Model
}

\author{
Zi-hui Zhang ${ }^{1} \quad$, Hui-nan $\mathrm{Zhu}^{2}$ ， Gang $\mathrm{He}^{3} \quad$ Xiu-fen Wang ${ }^{4 *}$ \\ 1 Basic Science Department, Tianjin Agricultural University \\ Tianjin 300384 China \\ 2 Humanities Department, Tianjin Agricultural University \\ Tianjin, 300384 , China \\ 3 Humanities Department, Tianjin Agricultural University \\ Tianjin, 300384 , China
}

4 Department of Computer Science and Information Engineering ,Tianjin Agricultural University, Tianjin, 300384 ,

${ }^{*}$ Corresponding author

\begin{abstract}
As the passenger throughput of airport is increasing, the security check of airport is confronting great pressure. In order to improve the efficiency of security check, this paper is to simulate the queuing process of security check through constructing cellular automaton model in order to get the indexes that influence security screening process. Here, we use cellular automaton to simulate the security screening process of different throughput. We can qualitatively determine the bottleneck of the whole process. As to the bottleneck, we can randomly shunt passengers who are going to enter the bottleneck to reduce congestion. Otherwise, different cultural backgrounds generate different social norms which show the time differences in security check. Therefore, we can divide passengers into 3 types and they would be shunted to 3 proprietary lanes. Later, after considering passenger composition type, the efficiency of airport staff, airlines' costs and so on, we give some proposals on how to optimize security screening process.
\end{abstract}

Key Words: passenger throughput, security screening process, cellular automaton model, passenger shunt

\section{Introduction}

After the September 11 attacks, the airport security checkpoints around the world are facing unprecedented pressure and challenges. It is still a difficult problem to balance relation between the requirements of passengers and the interests of airlines, even if the airport security condition has been significantly improved throughout the world.

According to the latest prediction data, the passenger throughput of Chinese airport will maintain an average annual growth rate of around $11.4 \%$. Furthermore, there will need 13 airports which annual passenger throughput exceeds 30 million, but now there are only 3 . And there will need 6 airports which annual passenger throughput around 20 million to 30 million, but now there are only 2 . Similarly, there will need 10 airports which annual passenger throughput around 10 million to 20 million, but now there are only $5^{[2]}$. From the above data, we derive that the passenger 
throughput will be more and more enormous. However, security checkpoint as an important part of affecting airport operation efficiency, if we can provide reliable solutions to reduce waiting time in this part, it will not only improve economic returns and security check management for airlines, but also decrease the flight delays and Improve passenger satisfaction. Therefore, it has very important practical

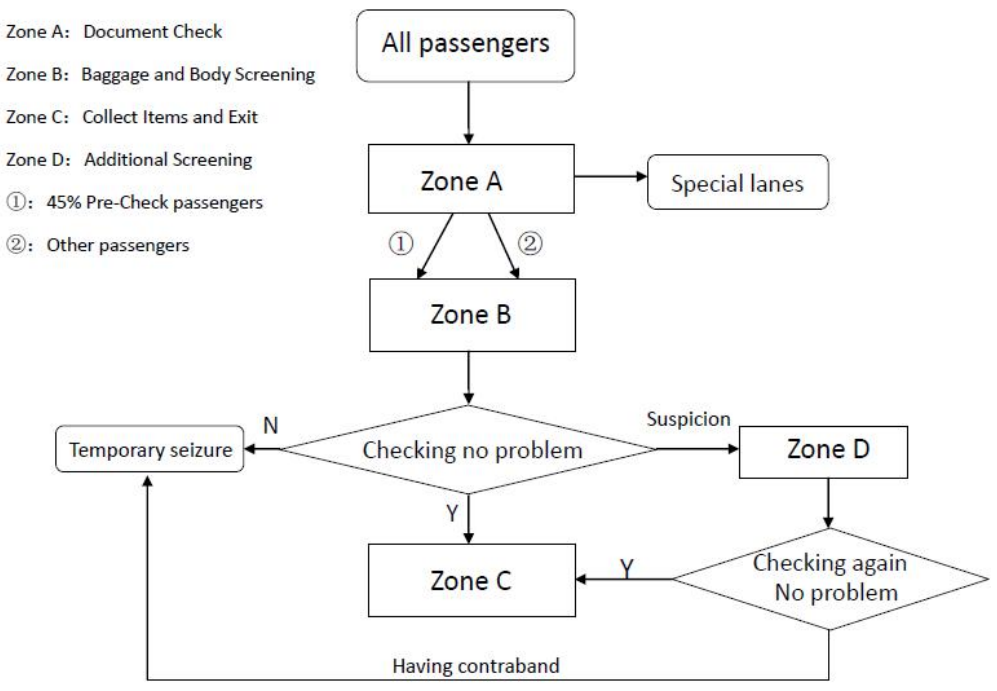

Figure 1: The change of passenger speed in single lane

1. We select passenger flow, density and speed in each zone as the data base to establish the passenger throughput simulation model via cellular automaton. Then explore the bottlenecks from these four zones.

2. With MATLAB software, we simulate the passenger screening processes in different passenger throughput. It can clearly show the congestions in each zone and the relations between passenger flow, density and speed. Then we can identify the bottlenecks.

3. After identifying the bottlenecks, we shunt passengers in corresponding zone to ease congestion for optimum process.

4. On the basis of shunt, we continue simulate the queuing process in Zone A and B via cellular automaton and get the variation diagrams of passenger flow, density and speed in these two zones.

5. We divide the passengers into three types according to the difference of screening time which caused by different cultural norms. Via cellular automaton model, we get passenger flow variation diagram which is based on the increased throughput of Zone A, B, C. solveing problem. consistent. maintain normal works. significance and application value to establish an optimum screening process which is suitable for most of airports around the world.

\section{Research Approaches}

To begin with, we analyze the security screening process and derive the results as shown in the following figure.

6. On the basis of above research results, after considering passenger type, airport officer efficiency and airlines' costs, we provide some proposals about security screening procedures and management policies.

\section{Research Assumptions}

We make the following assumptions about the

1. The screening speed of each security office is

2. All security checkpoint equipment always can

2. Ignore the influences to screening time caused by individual differences of age, gender and height.

4. No passengers jump in the queue.

5. There is no emergency for subjective reasons.

6. Passengers' documents are valid. Do not consider the situation of congestion caused by invalid documents. 


\section{Model building}

Considering the passengers in time and space both are discrete elements, passengers in security screening process have a finite number of state changes. The space and time range also are local factors. All these conditions are in accordance with the cellular automaton's discrete character both in time and space. Therefore, we choose cellular automaton model to simulate the state of passengers in security screening process.

\subsection{Model building and solution of question one}

In order to identify potential bottlenecks, we simulate the process of passenger security screening via cellular automaton model and select suitable indexes to measure the efficiency of each procedure. Then we gradually increase the passenger throughput to observe the variation of passenger flow, density and speed as the increase of passengers in each zone. From this, we can find out the deficiencies in security screening process and analyze the internal cause for the following improvement.

\subsubsection{Model building}

Via cellular automaton, we can simulate the passenger moving state. Thereby we define each passenger as a cellular length and determine the emergence time of passenger via Poisson distribution. Meanwhile, we determine several critical factors such as the queuing rules, the security screening time, screening rules and lanes in each zone. Specific rules are as follows:

1. The definition of passenger

Step1: the determination of seat array $\left\{\begin{array}{l}x, \text { stands for the abscissa where passenger is in } \\ y, \text { stands for the ordinate where passenger is in }\end{array}\right.$

A cellular represent a passenger.

Step2: the probability of passenger's emergence time

$$
P_{n}=\frac{\lambda^{n}}{n !} e^{-\lambda}, n=1,2, \ldots
$$

Step3: the definition of different passengers' probability
$\left\{\begin{array}{l}P_{d}, \text { stands for the probability of suspicious passengers } \\ P_{v} \text {, stands for probability probability of pre-passenger }\end{array}\right.$

From the problem description, we get $P_{t}=45 \%$

2. The definition of security screening

Step1: define the passenger screening position

$\left\{\begin{array}{l}x_{n}, \text { stands for the position where passenger is in } \\ y_{n}, \text { stands for the lane where passenger is in }\end{array}\right.$

Step2: determine the queuing discipline

$$
\left\{Z_{n}, \min \left(x_{n}-x_{n-1}\right)\right\}
$$

3. The determination of queuing

Step1: in order to prevent collision

$$
v=\min \left(0, v_{\max }, v_{\max } \geq 0\right)
$$

Step2: the update of position in moving

$$
x_{t+1}=x_{t}+v \times t
$$

Where $x_{t}$ stands for the position of passenger at time $t, v$ stands for the passengers moving speed. Step3: define the screening time $\left\{\begin{array}{l}T_{a}, \text { stands for the screening time in Zone } A \\ T_{b}, \text { stands for the screening time in Zone } B \\ T_{c}, \text { stands for the screening time in Zone } C\end{array}\right.$

\subsubsection{Model solution}

According to the above rules used to simulate passenger security screening process, we simulate the screening process for several times via cellular automaton model in MATLAB software as shown in Figure 2. In situations of different passenger throughput, we calculate the passenger flow, density and speed in each zone. Finally, we identify potential bottlenecks that disrupt passenger throughput through comparing the calculating results of the three indexes in each zone. 

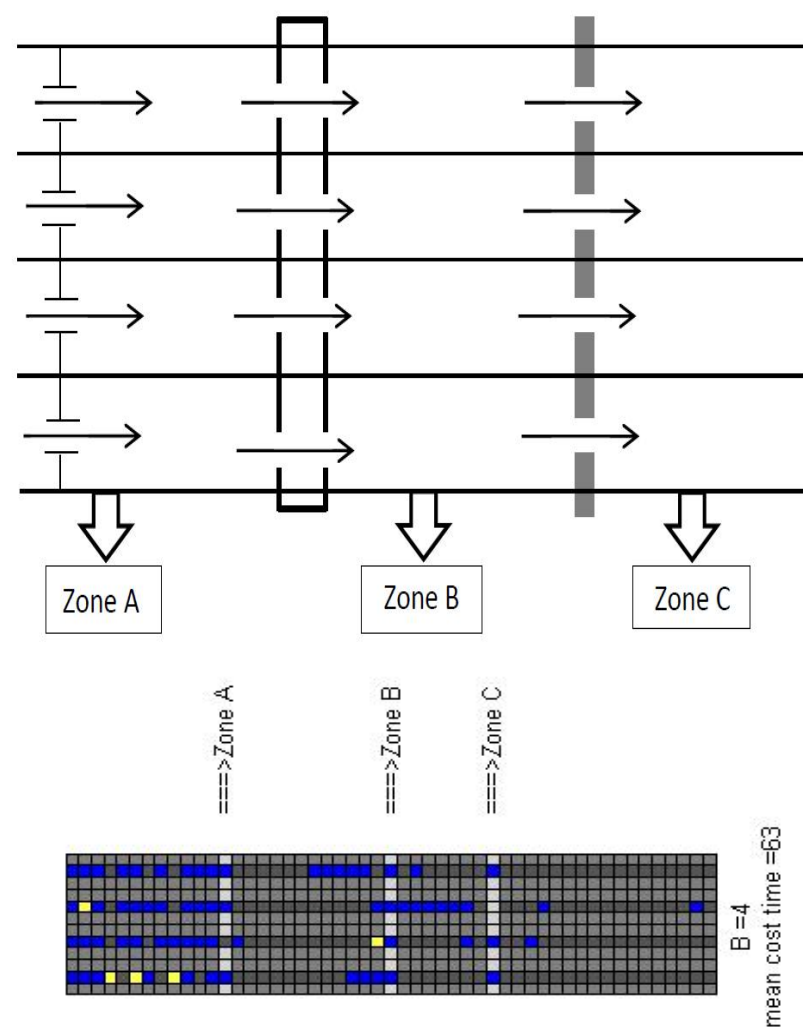

Figure 2: Illustration of four security screening lanes

Through the simulation in MATLAB software, we get the three variation diagrams of Zone A, B, C which is based on the change of passenger flow, density and speed in situations of different throughput. Because there is no congestion in pre-check lane shown via cellular automata simulation, we don't display the corresponding illustration in here.

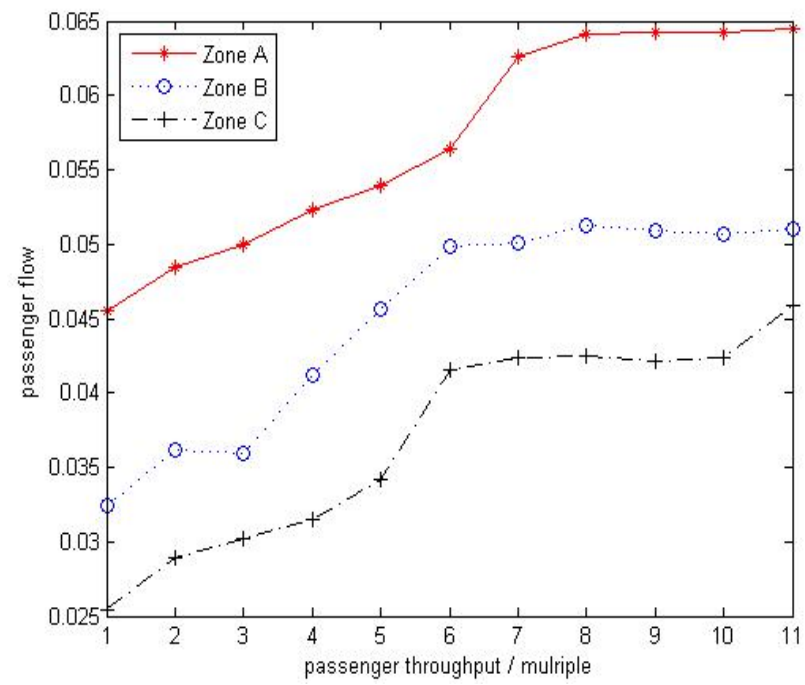

Figure 3: The change of passenger flow in single lane

As can be seen from Figure 3, as the increase of throughput, the passenger flow in Zone A, B, C both increase first and the remain constant. Whereas, passenger flow of Zone A changes after Zone B, but passenger flow in Zone $\mathrm{B}$ and $\mathrm{C}$ change almost synchronously.

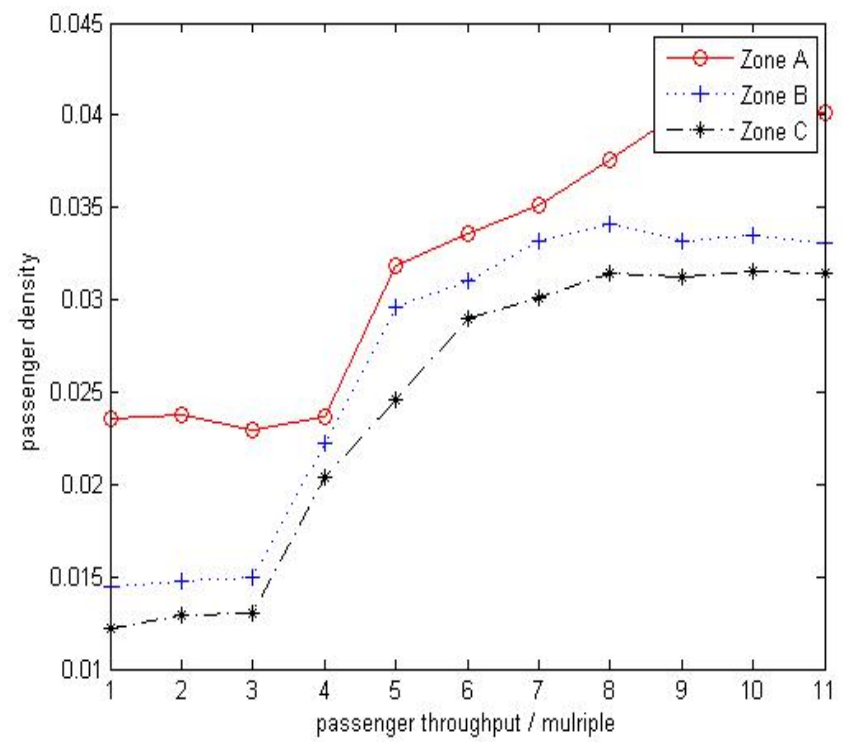

Figure 4: The change of passenger density in single lane

As we can see in Figure 4, with the increase of throughput, the passenger density in Zone A, B, C both change steady first and then increase, at last keep stable change again. The density in Zone $\mathrm{B}$ reaches the saturation point before the saturation point appears in Zone A.

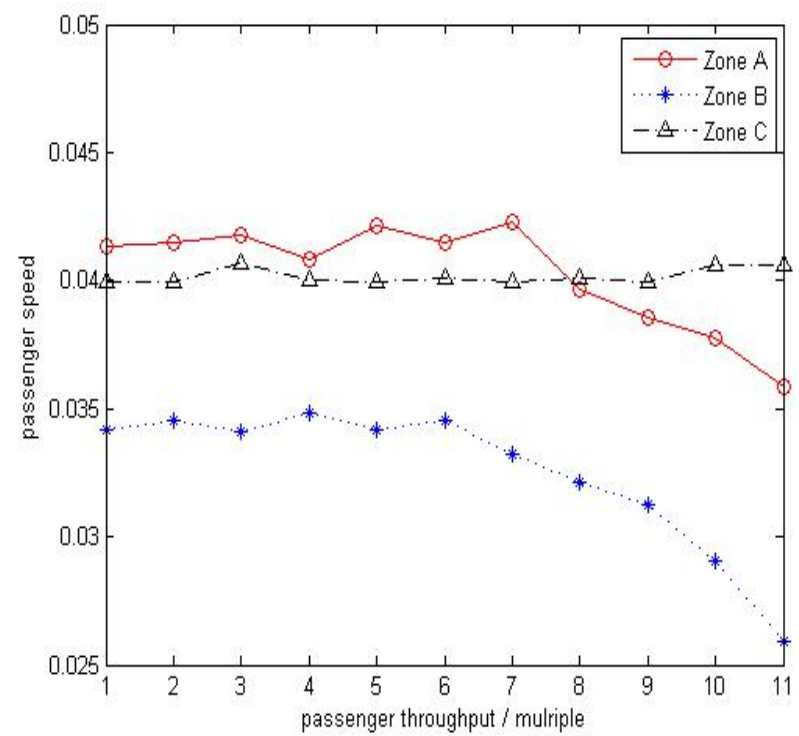

Figure 5: The change of passenger speed in single lane

As we can see in Figure 5, with the increase of throughput, the passenger speed in Zone $\mathrm{C}$ almost remains constant. The speed in Zone A and B both keep 
stable first, and then decrease. Moreover, the speed in Zone B decreases earlier than Zone A.

According to all above analysis, it shows that the passenger flow, density and speed in Zone B affect the change of these indexes in Zone A. The three indexes in Zone $\mathrm{A}$ and $\mathrm{C}$ are both higher than in Zone $\mathrm{C}$, also Zone $\mathrm{B}$ is located after Zone A and before Zone C. Thus, Zone $\mathrm{B}$ is the important factor of limiting the efficiency in Zone $\mathrm{A}$, and Zone $\mathrm{B}$ is the bottleneck of the whole security screening process.

\subsubsection{Model Test}

According to the data gived in this problem description, we can derive the screening time in each zone. Although these data are less, we still can calculate the number of passenger in each zone in a certain time range. Later, we compare this calculation results with the simulatin results of our .

Table 1: Passenger flow contrast table

\begin{tabular}{cccc}
\hline & $\begin{array}{c}\text { Real } \\
\text { passenger } \\
\text { traffic }\end{array}$ & $\begin{array}{c}\text { Simulation } \\
\text { of passenger } \\
\text { traffic }\end{array}$ & $\begin{array}{c}\text { Error } \\
\text { value }\end{array}$ \\
\hline Zone A & 538 & 516 & 4.00 \\
Zone B & 428 & 406 & 5.14 \\
Zone C & 418 & 400 & 4.31 \\
Zone D & 70 & 68 & 2.86 \\
\hline
\end{tabular}

As we can see in Table 1, in each security screening zone, the error value between actual passenger flow and the simulated passenger flow derived from cellular automaton model is less $6 \%$. Therefore, the simulation via cellular automaton model is reliable and the model is basically accurate.

\subsection{Model building and solution of question two}

\subsubsection{Model building}

Through the analysis of the first question, we identify that the bottleneck of the whole security screening process is in Zone B. It already appears congestion in Zone B when the passenger throughput is not big, so the key to reduce waiting time is to improve the passenger flow volume in Zone B. Hence we open two or more lanes to connect Zone A and Zone B, in order to optimize the security screening process.
Step1: define the diffluence after Zone A

$b_{1}$, stands for the first shunt lane from Zone B to Zone $C$ $b_{2}$, stands for the second shunt lane from Zone $B$ to Zone $C$

Step2: The position after passing Zone A

$$
\left\{x_{n}, \max \left(y_{a}-z_{n-1}, y_{a}-z_{n-1}\right)\right\}
$$

Resume that the passenger position, emergence time and moving speed are the same as in first question. Other conditions and rules equal, for Pre-Check lane, it is still a single lane and no shunt lane after Zone A.

\subsubsection{Model solution}

According to the above rules used to simulate passenger security screening process and the new rule of shunt in Zone B, we simulate the screening process for several times via cellular automaton model in MATLAB software as shown in Figure 6. In situations of different passenger throughput, we calculate the passenger flow, density and speed in each zone. At last, we compare the calculating results of the three indexes in each zone with the calculating results in first question, to see if there is an

improvement.
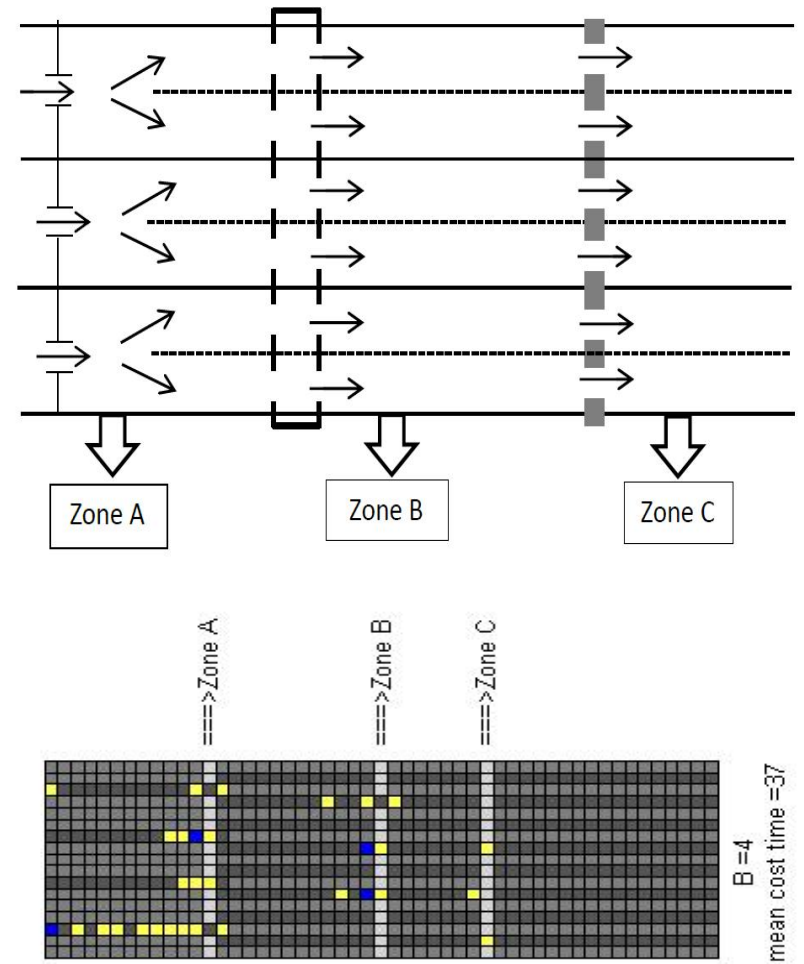

Figure 6: Illustration of security screening shunt lanes As we can see in Figure 7, under the pattern of 
shunt, as the increase of throughput, the passenger flow in Zone A and B both increase and the tendency of change are almost synchronous. Therefore, it is effective to randomly shunt passengers in two lanes after Zone A for the improvement of security screening efficiency. Also, this pattern helps us to solve the bottleneck of screening process.

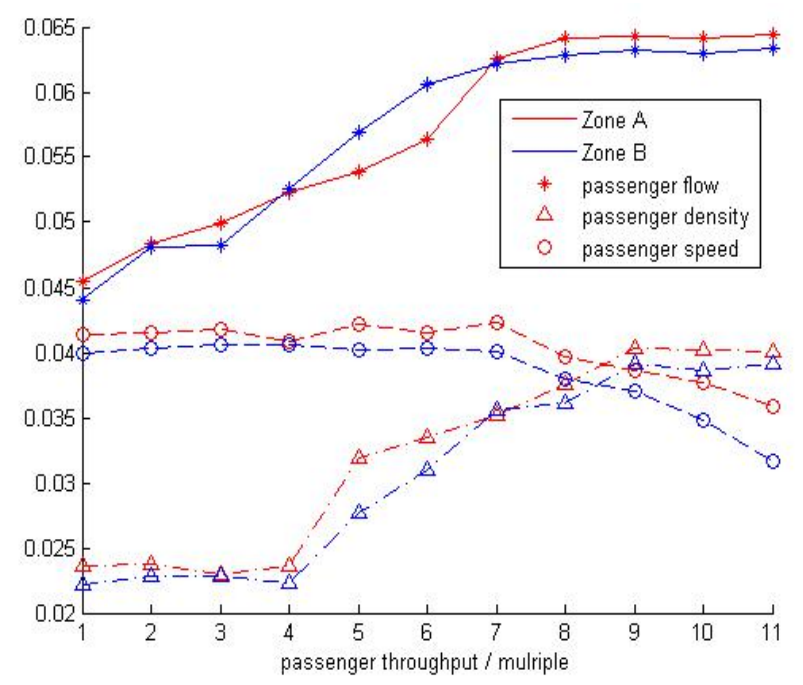

Figure 7: The variation diagram of each index in shunt situation

4.3 Model building and solution of question three

\subsubsection{Model building}

It is well known that different parts of the world have their own cultural norms that shape the local rules of social interaction. From the examples mentioned in task description, we know that it makes difference to screening time under the influence of regional differences. Therefore, we make simple classification on passenger according to the different screening time. And we refine the cellular automaton simulation model on the basis of the model in first question. As passenger throughput gradually increase, we observe the change of passenger flow, density and speed in each zone and compare with the observed results in second question, to see if there is an optimization.

Step1: define the passenger classification

\section{1, for the passenger who needs to be screened for long time $R_{w}=\{2$, for the passenger who needs to be screened for medium time \\ 3, for the passenger who needs to be screened for little time}

According to the shunt of screening time, we divide all passengers into three types and the emergence probability of each type is random. Every passenger can be the one of these three types.

Step2:define the emergence probability of different type passengers

$$
\left\{\begin{array}{l}
P_{1}, \text { stands for emergence probability of type } 1 \\
P_{2} \text {, stands for emergence probability of type } 2 \\
P_{3}, \text { stands for emergence probability of type } 3 \\
P_{1}+P_{2}+P_{3}=1
\end{array}\right.
$$

Passengers are divided into three types according to different screening times, there are fast passengers marked type 1 , medium speed passengers marked type 2 and slow passengers marked type3. After doing research, we know that their proportion is $2: 1: 2$, hence we make the flow chart of shunt and classification in security screening as shown in Figure 8. It sets specific shunt lanes for corresponding classification of passengers to improve the efficiency of whole security screening process and increase the passenger throughput in airports.

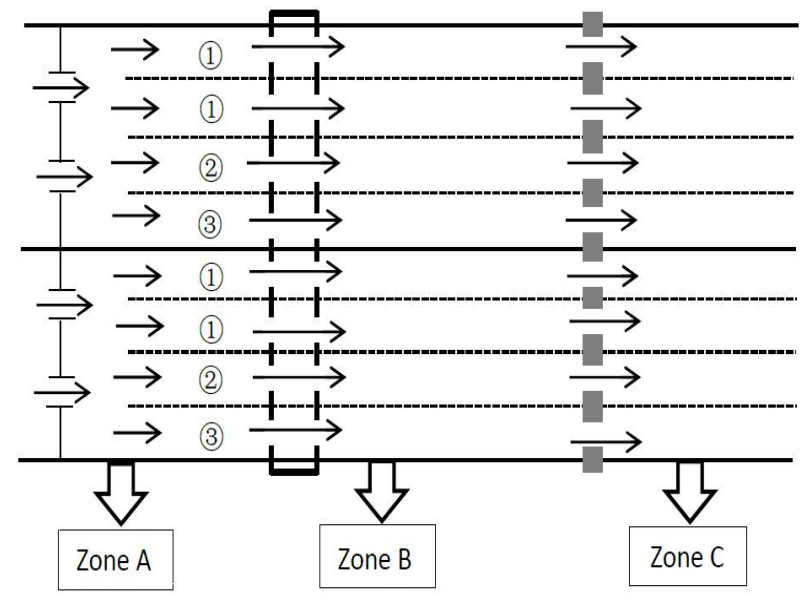

Figure 8: The flow chart of shunt and classification in security screening

\subsection{Model solution}

According to the above rules used to simulate passenger security screening process and the new rule of specific shunt, we simulate the screening process for several times via cellular automaton model in MATLAB software as shown in Figure 8. In situations of different passenger throughput, we calculate the passenger flow, density and speed in each zone. 


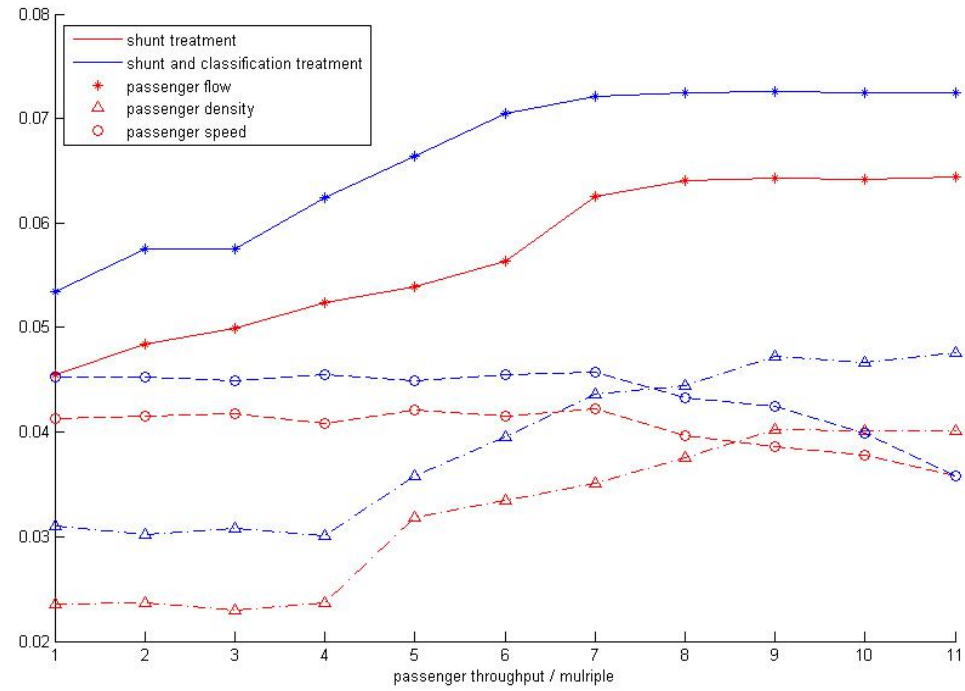

Figure 9: The variation of indexes in situation of shunt and classification

As we can see in Figure 9, as the increase of throughput, the passenger flow, density and speed are both bigger than those in the situation of only shunt and no classification. After shunting and then classify the passenger, it not only can eliminate the influence on security check caused by different social norms, but also can increase the passenger flow of the whole screening process to improve passenger throughput of the airports. It has a good effect on the development of the airport in the future.

\section{Policies and Recommendations}

According to the above results and the cellular automaton simulations in MATLAB, we can see that the bottleneck of the whole screening process is in Zone B. Although the Pre-Check program can alleviate this problem, it still cannot completely solve the bottleneck in Zone B. For the task b and task c, we choose the diffluence approach and the approach of passenger classification after diffluence, moreover, it can improve the screening efficiency of the whole security screening process through adopting these approaches. On the basis of the above research, we try to consider all aspects and make the following proposals for the security managers

1. Continue the implementation of Pre--Check program and increase its publicity. It can reduce the waiting time in Zone B to enhance the overall efficiency.

2. Investigate the current passenger throughput in airport and predict the future passenger throughput for better modifications.

3. Learn about the efficiency problem of airport security officers and try to facilitate communication among them.

4. Research the screening time of different passengers and make suitable classification for the diffluence security screening lanes.

5. Properly increase staffing and update screening machines when costs permit.

6 . Formulate reasonable policies like the child who is under 12 can become Pre-Check passenger for free.

The above proposals have been already combined with our research results. At last, we sincerely hope these proposals can help the optimization of airport security checkpoint.

\section{Conclution}

Airport is a bridge connecting the land traffic with the air traffic, and the airport security checkpoint is an important factor to its development. Especially for highly congested airports, the increasingly serious congestion and increasing flight delays cause the great loss to both passengers and airlines. Therefore, it requires airlines to continuously improve the security screening process and establish a safe, efficient and convenient checkpoint

The airport security screening model simulated by 
cellular automaton has high flexibility and universality. It can precisely simulate the each part of security screening process and make a comprehensive evaluation for the screening procedures in the absence of data. Eventually, we find out the bottleneck in security screening process through it. This model can clearly reveal the variation of passenger moving state. It is easy to set different parameters for the requirements of different airport situations, We do not use this model to consider more details due to the limited time. It can divide the passengers' security screening times into more time segments for better diffluence in future. The situation of some passengers queue together as a group can be considered in this model, such as the tourist groups and family members. And it will break the Poisson distribution. It can set more constraint conditions in specific airport, such as airport officers, screening costs, different throughput and so on.

Acknowledgements : Innovative training program for college students in Tianjin. Tianjin Agricultural University (201710061110)

\section{References}

[1]Lei Ju. Design and Implementation of Airport Security Information System [D].TianJin University.2013.5.(1).

[2]Lu Xun. Research on the Planning and Simulation of Passenger and Luggage Process at Airport Landside [D]. Nanjing University of Aeronautics and Astronautics.2008.6.(1).

[3]Liu Quan-ping. Study on Virtual Crowd Behaviors based on Multi-agent and Cellular Automat Model [D]. National University of Defense Technology.2013.11.(11)-(13).

[4]JI YunLI Hou-peng, FU Xin-yu. Analysis of Airport Passenger Flow Quantity Based on Compound Poisson Process [J]. Chongqing Technol Business Univ. ( Nat Sci Ed), Vol.30 NO.5

[5]JIAO Peng-peng. Forecasting Method and Its Mechanism of Impacts on A irport Passenger Throughput [J]. Journal ofTransportation System s Engineering and Information Technology,2005.5: Vol.5 No.1 